\title{
Contrast Sensitivity and Visual Acuity under Low Light Conditions in Macular Telangiectasia Type 2
}

\author{
*Simone Müller, $\mathrm{MD}^{1}$ \\ *Tjebo FC Heeren, $\mathrm{MD}^{1,2}$ \\ Roberto Bonelli, ${ }^{3}$ \\ Marcus Fruttiger, ${ }^{4}$ \\ Peter Charbel Issa, MD, Dphil ${ }^{5}$ \\ Catherine Egan, MD, FRANZCO 2 \\ Frank G Holz, MD ${ }^{1}$
}

*SM and TFCH contributed equally to this manuscript

1. University Eye Hospital Bonn, Bonn, Germany

2. Moorfields Eye Hospital NHS Foundation Trust, London, UK

3. The Walter and Eliza Hall Institute of Medical Research and the department of Medical Biology, University of Melbourne, Parkville, Victoria, Australia

4. Institute of Ophthalmology, University College London, London, UK

5. Oxford Eye Hospital, OUH NHS Foundation Trust and the Nuffield Laboratory of Ophthalmology, Department of Clinical Neurosciences, University of Oxford

Manuscript word count: 2532 words

\section{Corresponding author}

Tjebo FC Heeren, MD

Moorfields Eye Hospital NHS Foundation Trust

162 City Road, London, EC1V 2PD

Email: Tjebo.Heeren@moorfields.nhs.uk

Phone: 00442075662815

Financial support: Lowy Medical Research Institute. This work was also made possible through the Victorian State Government Operational Infrastructure Support and Australian Government National Health and Medical Research Council (NHMRC) independent research Institute Infrastructure Support Scheme (IRIISS). R. Bonelli was supported by the Melbourne International Research Scholarship. The funding organization had no role in the design or conduct of this research. Catherine Egan is funded or partially funded by the National Institute of health research (NIHR) Biomedical Research Centre for Ophthalmology, Moorfields Eye Hospital NHS Foundation Trust, London, United Kingdom.

Conflict of interest: No conflicting relationships exist for any authors. 
Author contributorship statement:

$\mathrm{SM}$ and TFCH:

conception and design of the work, data acquisition, analysis and interpretation of data. Drafting the work and revising it critically for important intellectual content.

RB: Analysis and interpretation of data, critical revision of the manuscript

$\mathrm{MF}, \mathrm{PCl}, \mathrm{CE}, \mathrm{FH}$ : Interpretation of data, critical revision of the manuscript 


\section{Synopsis/Precis 35 words}

Contrast sensitivity is considerably impaired under low light conditions in early disease stages of MacTel. It might aid earlier detection of the condition and also be useful as an additional outcome measure in interventional trials. 


\section{ABSTRACT}

\section{Background/Aims}

Macular pigment optical density (MPOD) is centrally depleted early on in macular telangiectasia type 2 (MacTel). Contrast sensitivity might be related to MPOD. Therefore, CS might be impaired in early MacTel. The effect of low luminance was assessed on both CS and best corrected visual acuity (BCVA).

\section{Methods}

Cross-sectional study. Pelli-Robson charts were used for CS testing at $1 \mathrm{~m}$ in photopic (110 lux) and mesopic (1 lux) conditions. BCVA was tested with ETDRS charts, low luminance visual acuity (LLVA) with a 2.0-log unit neutral density filter. MPOD was obtained with dual wavelength autofluorescence.

\section{Results}

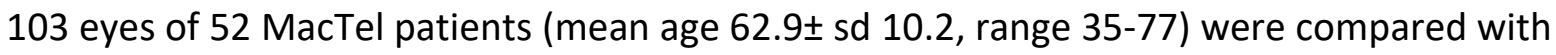
34 healthy eyes of 17 controls (mean age $65.2 \pm$ sd 7.4, range 53-78). CS was significantly lower in eyes with MacTel. This impairment was higher in low light conditions (LL-CS). Eyes with early MacTel stages had significantly lower LL-CS than controls, but normal (photopic) CS. The results were similar but less pronounced for BCVA/LLVA. Decrease of CS was correlated with loss of MPOD.

\section{Conclusions}

Low light conditions have a detrimental effect on visual performance in MacTel. Impaired CS might correlate to MPOD depletion as a pathognomonic finding in MacTel. Functional impairment might precede structural disintegration, indicating dysfunction on a cellular level. The applied tests might be useful as additional functional assessments in clinical routine and as outcome measures in future interventional clinical trials. 


\section{INTRODUCTION}

Macular telangiectasia type 2 (MacTel) is a bilateral disease with characteristic clinical features in funduscopy, fluorescein angiography, fundus autofluorescence, and spectral domain optical coherence tomography (SD-OCT). ${ }^{1}$ Visual symptoms typically start in the fifth and sixth decade of life, usually with reading problems and/or metamorphopsia. ${ }^{2-4}$ The impaired reading performance, often despite well preserved central visual acuity, may be explained by compromised and progressive paracentral retinal dysfunction with characteristic paracentral scotomata. ${ }^{5-7}$

Recently, investigational clinical trials have been initiated. An intraocular implant with ciliary neurotrophic factor (CNTF) secretion has been used in a phase-2-trial that has recently been completed. In this trial, the loss of ellipsoid zone on OCT imaging is used as a surrogate measure for disease progression as it has been shown to correlate well with functional loss in microperimetry. ${ }^{8,9}$ However, potential routine clinical outcome measures of macular function other than visual acuity and microperimetry testing, such as contrast sensitivity and low luminance visual acuity, have not yet been explored in detail.

The most consistent finding in eyes with MacTel is the loss of macular luteal pigments. ${ }^{10,11}$ Although the function of macular pigment is debated, there is a general consensus that it reduces light scatter in green/blueish light conditions due to its absorption maximum in the blue spectrum. ${ }^{12}$ This is thought to improve contrast sensitivity, and indeed some studies indicate an association of macular pigment optical density (MPOD) and contrast sensitivity: Recently, two prospective trials with macular pigment supplementation showed a statistically significant improvement of contrast sensitivity of lower to medium spatial frequencies with increasing macular pigment (MP) levels. ${ }^{13,14}$ 
A potential association between macular pigment density and contrast sensitivity has not yet been investigated in MacTel.

We hypothesized that that contrast sensitivity and low light visual acuity are disturbed earlier than best corrected visual acuity. Secondly, we hypothesized that the reduced macular pigment density in patients with MacTel would result in reduced contrast sensitivity. Using simple, reliable and cost-effective methods such as ETDRS and PelliRobson charts, our intention was to investigate if those tests might be useful as additional clinical endpoints in future interventional trials. 


\section{METHODS}

\section{Patients and Staging}

Patients of the MacTel Natural History and Observation Study (NHOS) were examined in the University Eye Hospital Bonn, Germany. The study was conducted in accordance with the Declaration of Helsinki, institutional review board (IRB) approval had been obtained, and informed consent was obtained from all participants. Eyes were divided into eyes with visible morphological changes in any imaging modality and eyes without any changes ('stage 0 '). Naturally, it was only possible to classify an eye as 'stage 0 ' in the presence of a definite MacTel diagnosis in the fellow eye-this was based on the assumption that MacTel is a bilateral disease. Controls were age matched and had no evidence or history of retinal diseases.

\section{Contrast sensitivity}

Monocular contrast sensitivity testing was performed on both eyes with Pelli Robson Letter charts at a test distance of $1 \mathrm{~m}$ with added correction for near distance according to the manufacturer's instructions. ${ }^{15}$ Testing was performed under both photopic and mesopic light conditions (mean room illumination set at 110 lux and 1.0 lux, respectively). Illumination was determined at several locations within the consultation room using a calibrated Light Meter Lux 840021 (Sper Scientific, Scottsdale, USA). Individuals underwent 10 minutes of light adaptation in the respective light conditions prior to testing. Two different test charts were used in alternating manner, and mesopic testing was conducted prior to photopic testing, in order to reduce memorisation effects. The contrast sensitivity score was calculated as proposed by Pelli and Robson, and therefore steps of 0.15 log units were obtained. Measurement of contrast sensitivity under low light conditions were termed 
'low light contrast sensitivity' (LLCS) as opposed to those under photopic conditions (CS). The relative deficit between CS and LLCS was termed 'low luminance deficit of contrast sensitivity' (LLD-CS).

\section{Low luminance deficit}

Monocular BCVA and low luminance visual acuity (LLVA) were measured in both eyes at a test distance of $4 \mathrm{~m}$ by standard Early Treatment of Diabetic Retinopathy Study (ETDRS) protocols. LLVA was measured and analyzed according to Sunness et al., ${ }^{16}$ using a $2.0-\log$ unit neutral density filter placed in front of each eye, using the same ETDRS charts. Low luminance deficit of visual acuity (LLD-VA) was defined as the difference between LLVA and BCVA. BCVA, LLVA and LLD-VA were expressed in logMAR.

\section{Macular pigment optical density}

Macular pigment optical density (MPOD) was measured using dual wavelength autofluorescence using the HRA Spectralis (Heidelberg Engineering, Heidelberg, Germany) and the corresponding software (HEYEX, version 1.9.10.0, HRA/Spectralis Viewing Module 6.0.13.0). A four second video of simultaneous green and blue autofluorescence was obtained and then the MPOD map created following the manufacturer's standard of procedure. Loss of MPOD was graded into three classes of increasing MPOD loss, suggested by Zeimer et al. ${ }^{17}$ In brief, class 1 showed MPOD loss limited to the temporal macula; in class 2, MPOD loss affected the foveola and extended into the nasal part of the macula; class 3 was defined as a complete MPOD loss with or without a ring of increased MPOD at 5 to 7 eccentricity. 


\section{Statistical analysis}

Statistical significance was assessed using linear mixed models. Each model included a random intercept to take into account the correlation within subject observations as well as a fixed term to take into account potential differences between right and left eyes. All statistical analyses were performed using the R statistical software (R development Core Team, Vienna, Austria). All statistical results from the linear mixed model are presented by showing the fixed effect term ' $b$ ' which indicates the mean increment in the studied phenomenon for a unit change in the the condition of interest. A p-value $<0.05$ was considered as significant. The 2D-density contour-plots were calculated with the 2D-kernel density estimation function $k d e 2 d$ from the MASS package in R (version 3.3.1).

\section{RESULTS}

Out of 122 eligible participants of the NHOS, we were able to recruit 52 patients (mean age $62.9 \pm$ sd 10.2, range 35-77). The control cohort consisted of 34 eyes of 17 controls (mean age $65.2 \pm$ sd 7.4, range 53-78). Contrast sensitivity

One eye of a MacTel patient was excluded from contrast sensitivity and visual acuity analysis because of a non-plausible test result (increase of contrast sensitivity under mesopic conditions). Table 1 summarises the results of contrast sensitivity and visual acuity testing. The remaining 103 eyes with MacTel had significantly impaired photopic and mesopic contrast sensitivity when compared to controls $(b=-0.237, p$-value $<0.001$ and $b=-$ $0.373, p$-value $<0.001)$. This impairment was higher in mesopic condition when compared with photopic conditions $(b=-0.136, p$-value=0.003), shown in figure 1 . Eyes with higher or 
normal photopic contrast sensitivity had a pronounced and significantly higher impairment of contrast sensitivity in mesopic conditions when compared with controls $(b=-0.259, p-$ value<0.001). 'Stage 0 ' eyes $(n=6)$ were within this group (figure 1 , crosses).

MacTel eyes showed a significantly decreased BCVA $(b=0.2801, p$-value $<0.001)$ and LLVA (0.346, p-value<0.001). BCVA was significantly associated with CS and LL-CS (figure 3, left panel, $b=-0.356, p$-value $<0.001$ and $b=-0.275, p$-value $<0.001)$.

LLD-VA was significantly higher in MacTel eyes when compared with controls $(b=0.065, p$ value $=0.006$ ). However, the absolute differences were not very pronounced. Figure 2 shows the normalized loss of function under low light/ low luminance conditions for both contrast sensitivity (LLD-CS) and visual acuity (LLD-VA). Macular pigment optical density (MPOD) MPOD measurement was discarded in two eyesdue to poor quality MPOD images. Of the remaining 102 eyes, 6 eyes (6\%) were classified as MPOD class 0, lacking any visible MPOD reduction. 17 eyes (17\%) as class 1, 33 eyes (32\%) as class 2 and 46 eyes (45\%) as class 3. Figure 3 shows the contrast sensitivity of eyes with different classes of macular pigment loss. Mesopic contrast sensitivity was similarly impaired in all eyes. Photopic contrast sensitivity decreased with higher MPOD classes: We found a significant decrement in contrast sensitivity in photopic condition for each unit increment in MPOD class $(b=-0.068$, $p$-value $<0.001)$. Although present, this decrement was not significant in mesopic light condition $(b=-0.025, p$-value=0.196). Importantly, significance of MPOD classes on contrast sensitivity vanished completely when correcting for BCVA indicating that the effect of MPOD 
on contrast sensitivity in photopic condition might be connected to its correlation with BCVA. 


\section{DISCUSSION}

Summarizing the above results, eyes with MacTel showed a generalized impairment of contrast sensitivity which increased in low light conditions (increased LLD-CS). This was found in eyes without detected morphological changes, and in eyes with normal BCVA and no absolute scotoma in microperimetry testing. A similar, but markedly less pronounced effect was found with LLD-VA.

Our results indicate that low light has a detrimental effect on visual function in MacTel. This is detectable as significant impairment of contrast sensitivity and increased low luminance deficit when compared with healthy eyes. This effect is most pronounced in early disease stages: All eyes (early and late stages) seem to have similarly low levels of mesopic contrast sensitivity. While photopic contrast sensitivity and BCVA is normal in eyes with early disease stages, it is already impaired in eyes with later disease stages. Thus, in our figures 2 to 4 , eyes with early stages are found rather in the bottom left of the graphs, whereas eyes with late stages are more towards the top right. An increased impairment of contrast sensitivity under mesopic conditions (increased LLD-CS) has been shown in other macular diseases including AMD and diabetic retinopathy as well as in neurological disorders such as Parkinson disease and multiple sclerosis (reviewed in ${ }^{18}$ ). More importantly, we found a lower (mesopic) contrast sensitivity in eyes without visible structural changes, pointing towards cellular stress on a micro-cellular level (e.g., impaired metabolism), ultimately leading to structural changes (and in the end cell atrophy). Impaired contrast sensitivity without morphological changes was also found in patients with diabetes. ${ }^{19}$ Interestingly, diabetes seems to occur more frequently in patients with MacTel, ${ }^{1}$ hinting towards common metabolic dysregulations at least in some patients - likewise leading to similar 
functional impairments. We were surprised by the degree of contrast sensitivity impairment in seemingly unaffected MacTel eyes. Charbel Issa and Heeren have recently shown that those eyes revealed a reduced Stiles-Crawford-effect. ${ }^{20}$ Cone misalignment might cause metabolic stress, as recently hypothesized by Eckmiller ${ }^{21}$ and metabolic changes might impair contrast sensitivity. ${ }^{22}$ However, the implications of these findings still need further investigations.

The difference between MacTel eyes and controls was less pronounced in low luminance visual acuity testing. As both groups showed a marked overlap, LLD-VAmight not be useful in order to differentiate between affected and non-affected eyes. We can only speculate why the LLD-VA is less markedly increased than LLD-CS. A possible explanation might be that visual acuity testing is based on high spatial frequencies with maximum contrast (> $85 \%)$, and therefore can be considered a special case of contrast testing. Even when the luminance of both background and stimuli are reduced, those letters still yield a rather high contrast, and therefore the effect of visual impairment might not be so pronounced. A preceding loss of rod function ${ }^{10,23}$ might also contribute to impaired contrast sensitivity in mesopic condition, especially in the spatial range of Pelli-Robson letters that overlap with the range of maximum rod contrast sensitivity. ${ }^{24}$ Further studies are needed to specify the affection of contrast sensitivity in different spatial frequencies, especially under mesopic conditions.

Our study showed a cross sectional correlation between the pattern of macular pigment loss and contrast sensitivity. Although a longitudinal study would be preferable to show correlations in changes of both, this is very challenging in reality. First, the disease is only very slowly progressing and there is currently not much data available about the 
longitudinal natural history of macular pigment density. In fact, to our best knowledge there is currently no data published on the change of macular pigment patterns over time. Secondly, supplementation with Lutein and Zeaxanthin showed no change of central accumulation in two independent studies. ${ }^{17,25}$ It is not surprising that Choi et al were not able to find a correlation of contrast sensitivity and macular pigment in their study which only included eyes with complete loss of central macular pigment ('MPOD class 3'). Although MP supplementation was shown to increase the optical density at 5-7 degree eccentricity, this change is unlikely to influence contrast sensitivity, as the latter is usually tested within the central 10 degrees and not beyond. On this background, a cross sectional approach in order to test for correlation between contrast sensitivity seems a viable option in our opinion. . However, it is possible that there might be another factor which leads to decreased luteal pigment, BCVA and contrast sensitivity alike.

Potential limitations of our study include the use of a categorical variable for MPOD. A continuous variable would certainly have been preferable for the correlation analysis with contrast sensitivity and BCVA. The reason for using (categorical) MPOD patterns of increasing macular pigment loss instead was the problematic and rather unreliable quantification of MPOD based on the images. The method, as good as it is, is very dependent on factors such as retinal illumination, eye movements during image acquisition and lens opacity and the generalized macular pigment density in the more peripheral retina. This led to not entirely plausible large ranges of MPOD in our cohort. On the other hand, we have observed that the patterns of macular pigment loss were very reproducible (data not shown). 
A smaller limitation of our study is the limited number of available Pelli-Robson charts which

might have led to a memorizing effect. We tried to reduce this bias by alternating and

testing first under mesopic and then under photopic conditions.

Also, the statistical power is only limited towards the six eyes with 'unilateral' MacTel (stage

0). Unfortunately, not many of those cases have been identified yet, and our results might

be affected by small numbers. We are currently continuing to identify further unilateral

cases in order to validate our results and gain further insights.

Overall, our results underscore the importance of background light levels for the visual performance in patients with MacTel. Their contrast perception is impaired, and even more so in low light levels (simulation in Figure 4). The impairment of contrast vision under reduced light conditions might be correlated to loss of macular pigment optical density as a pathognomonic finding in MacTel. Our results suggest that even eyes without impairment in visual acuity testing and/or without morphological changes might already show impaired contrast sensitivity. Although we were not able to pinpoint the functional loss to a particular structural or cellular dysfunction, the applied tests have the advantage to be well established, cost-effective, quick and easy to perform. They would, therefore, qualify to be used as additional functional assessments both in clinical routine and potentially as outcome measures in future interventional clinical trials. 
Table 1: Summary of results

\begin{tabular}{|l|l|l|}
\hline Measure & $\begin{array}{l}\text { Controls } \\
\mathrm{n}=34\end{array}$ & $\begin{array}{l}\text { MacTel } \\
\mathrm{n}=103^{*}\end{array}$ \\
\hline 'Photopic' contrast sensitivity (CS) & $1.87 \pm 0.13$ & $1.63 \pm 0.22$ \\
\hline 'Mesopic' contrast sensitivity (LL-CS) & $1.58 \pm 0.13$ & $1.21 \pm 0.19$ \\
\hline $\begin{array}{l}\text { Low luminance deficit of contrast sensitivity } \\
\text { (LLD-CS) }\end{array}$ & $0.28 \pm 0.11$ & $0.42 \pm 0.19$ \\
\hline Best corrected visual acuity (BCVA), logMAR & $-0.04 \pm 0.08$ & $0.25 \pm 0.28$ \\
\hline Low luminance visual acuity, logMAR & $0.16 \pm 0.12$ & $0.51 \pm 0.26$ \\
\hline Low luminance deficit of visual acuity (LLD-VA) & $0.20 \pm 0.07$ & $0.26 \pm 0.11$ \\
\hline
\end{tabular}

* one eye was removed from analysis because of non-plausible test results (increase of contrast sensitivity in low luminance conditions). $p$-Value $<0.001$ for all measures except for LLD-VA ( $p$-value $=0.006)$ 


\section{Figure 1:}

Every dot represents an individual eye, jitter was applied in order to show every data point. The density contour lines show the estimated density distribution. The y-axis shows the loss of contrast sensitivity (CS) and best corrected visual acuity (BCVA) in low light/ low luminance conditions (LLDCS/ LLD-VA). It is expected that all eyes have an impaired CS/VA under low light conditions. Controls and MacTel eyes do hardly overlap, meaning a relatively higher functional impairment for MacTel eyes when compared with controls, even when photopic contrast sensitivity is normal.

\section{Figure 2:}

Low luminance deficit of contrast sensitivity (LLD-CS) and low luminance deficit of visual acuity (LLDVA). In both cases, there is an increasing loss of function with decreasing best corrected visual acuity (BCVA). The figures show normalized values (z-scores), based on the mean/sd of the full sample (controls and MacTel eyes). Eyes with MacTel and normal BCVA showed both higher LLD-CS and higher LLD-VA than healthy control eyes. However, this effect was more pronounced for LLD-CS: The probability of having higher LLD than controls is higher for CS than for VA. (The density contours show the estimates of the probability of distribution) Eyes with stage 0 disease (crosses) had markedly increased LLD-CS, but less clearly increased LLD-VA when compared with controls.

\section{Figure 3}

Blue circles represent one individual eye with MacTel (jitter was applied). Density contour lines show the estimated density distribution. The red transparent dot shows the point with the highest density of healthy control eyes as shown in figure 1 . The higher the class of macular pigment optical density (MPOD) the lower was photopic contrast sensitivity. Loss of contrast sensitivity, however, showed a tendency to become lower with increasing classes, indicating that mesopic contrast sensitivity is impaired early on and also indicating a possible 'floor effect' of mesopic contrast sensitivity in MacTel. 


\section{Figure 4}

Simulation of contrast vision for MacTel patients. We do not see a structural difference between the healthy fundus on the left, and the seemingly 'unaffected' fellow eye of a patient with macular telangiectasia type 2 (MacTel) on the right. The macular pigment measurement of this eye can be seen in Figure 3 (example image for 'class 0'). Mesopic contrast sensitivity of this eye was almost $50 \%$ lower than the mean mesopic contrast sensitivity of our controls. This difference of contrast perception is simulated for photopic conditions (upper row) and mesopic conditions (lower row). A stimulus that is fairly easily perceptible with healthy eyes, is hardly visible for eyes with MacTel. The bottom right shows a contrast of $4.6 \%$, which was the contrast threshold of this eye (log contrast sensitivity 1.35). Mean contrast threshold in controls in mesopic condition was around $2.5 \%$ (log contrast sensitivity 1.6). The simulation is based on the simplifying assumption that the perception of contrast differences behaves proportional throughout all contrast ranges. 


\section{REFERENCES}

1. Charbel Issa P, Gillies MC, Chew EY, et al. Macular telangiectasia type 2. Prog Retin Eye Res 2013; 34: 49-77.

2. Heeren TF, Holz FG, Charbel Issa P. First symptoms and their age of onset in macular telangiectasia type 2. Retina 2014; 34(5): 916-9.

3. Charbel Issa P, Holz FG, Scholl HP. Metamorphopsia in patients with macular telangiectasia type 2. Documenta ophthalmologica Advances in ophthalmology 2009; 119(2): 133-40.

4. Finger RP, Charbel Issa P, Fimmers R, Holz FG, Rubin GS, Scholl HP. Reading performance is reduced by parafoveal scotomas in patients with macular telangiectasia type 2. Invest Ophthalmol Vis Sci 2009; 50(3): 1366-70.

5. Heeren TF, Clemons T, Scholl HP, Bird AC, Holz FG, Charbel Issa P. Progression of Vision Loss in Macular Telangiectasia Type 2. Invest Ophthalmol Vis Sci 2015; 56(6): 3905-12.

6. Vujosevic $S$, Heeren TFC, Florea D, et al. SCOTOMA CHARACTERISTICS IN MACULAR TELANGIECTASIA TYPE 2: MacTel Project Report No. 7-The MacTel Research Group. Retina 2018; 38 Suppl 1: S14-s9.

7. Charbel Issa P, Helb HM, Rohrschneider K, Holz FG, Scholl HP. Microperimetric assessment of patients with type 2 idiopathic macular telangiectasia. Invest Ophthalmol Vis Sci 2007; 48(8): 378895.

8. Heeren TFC, Kitka D, Florea D, et al. LONGITUDINAL CORRELATION OF ELLIPSOID ZONE LOSS AND FUNCTIONAL LOSS IN MACULAR TELANGIECTASIA TYPE 2. Retina 2017.

9. Sallo FB, Peto T, Egan C, et al. The IS/OS junction layer in the natural history of type 2 idiopathic macular telangiectasia. Invest Ophthalmol Vis Sci 2012; 53(12): 7889-95.

10. Powner MB, Gillies MC, Zhu M, Vevis K, Hunyor AP, Fruttiger M. Loss of Muller's cells and photoreceptors in macular telangiectasia type 2. Ophthalmology 2013; 120(11): 2344-52.

11. Charbel Issa P, Berendschot TT, Staurenghi G, Holz FG, Scholl HP. Confocal blue reflectance imaging in type 2 idiopathic macular telangiectasia. Invest Ophthalmol Vis Sci 2008; 49(3): 1172-7.

12. Snodderly DM, Brown PK, Delori FC, Auran JD. The macular pigment. I. Absorbance spectra, localization, and discrimination from other yellow pigments in primate retinas. Invest Ophthalmol Vis Sci 1984; 25(6): 660-73.

13. Nolan JM, Loughman J, Akkali MC, et al. The impact of macular pigment augmentation on visual performance in normal subjects: COMPASS. Vision Res 2011; 51(5): 459-69.

14. Nolan JM, Power R, Stringham J, et al. Enrichment of Macular Pigment Enhances Contrast Sensitivity in Subjects Free of Retinal Disease: Central Retinal Enrichment Supplementation Trials Report 1Central Retinal Enrichment Supplementation Trials. Investigative Ophthalmology \& Visual Science 2016; 57(7): 3429-39.

15. Pelli D, Robson J, Wilkins A. The design of a new letter chart for measuring contrast sensitivity. Clinical Vision Sciences 1988; 2(3): 187-99.

16. Sunness JS, Rubin GS, Broman A, Applegate CA, Bressler NM, Hawkins BS. Low luminance visual dysfunction as a predictor of subsequent visual acuity loss from geographic atrophy in agerelated macular degeneration. Ophthalmology 2008; 115(9): 1480-8, 8.e1-2.

17. Zeimer MB, Kromer I, Spital G, Lommatzsch A, Pauleikhoff D. Macular telangiectasia: patterns of distribution of macular pigment and response to supplementation. Retina 2010; 30(8): 1282-93.

18. Pelli DG, Bex P. Measuring contrast sensitivity. Vision Res 2013; 90: 10-4.

19. Della Sala S, Bertoni G, Somazzi L, Stubbe F, Wilkins AJ. Impaired contrast sensitivity in diabetic patients with and without retinopathy: a new technique for rapid assessment. $\mathrm{Br} J$ Ophthalmol 1985; 69(2): 136-42.

20. Charbel Issa P, Heeren TF, Kupitz EH, Holz FG, Berendschot TT. VERY EARLY DISEASE MANIFESTATIONS OF MACULAR TELANGIECTASIA TYPE 2. Retina 2016; 36(3): 524-34. 
21. Eckmiller MS. Defective cone photoreceptor cytoskeleton, alignment, feedback, and energetics can lead to energy depletion in macular degeneration. Prog Retin Eye Res 2004; 23(5): 495-522.

22. Barlow RB, Khan M, Farell B. Metabolic Modulation of Human Visual Sensitivity. Investigative Ophthalmology \& Visual Science 2003; 44(13): 2708-.

23. Schmitz-Valckenberg S, Fan K, Nugent A, et al. Correlation of functional impairment and morphological alterations in patients with group $2 \mathrm{~A}$ idiopathic juxtafoveal retinal telangiectasia. Archives of ophthalmology (Chicago, III : 1960) 2008; 126(3): 330-5.

24. Hess RF, Nordby K, Pointer JS. Regional variation of contrast sensitivity across the retina of the achromat: sensitivity of human rod vision. The Journal of physiology 1987; 388: 101-19.

25. Choi RY, Gorusupudi A, Wegner K, Sharifzadeh M, Gellermann W, Bernstein PS. MACULAR PIGMENT DISTRIBUTION RESPONSES TO HIGH-DOSE ZEAXANTHIN SUPPLEMENTATION IN PATIENTS WITH MACULAR TELANGIECTASIA TYPE 2. Retina 2017; 37(12): 2238-47. 